Draft VERSion June 22, 2021

Preprint typeset using $\mathrm{IAT}_{\mathrm{E}} \mathrm{X}$ style emulateapj v. 5/2/11

\title{
TEMPERATURE FLUCTUATIONS DRIVEN BY MAGNETOROTATIONAL INSTABILITY IN PROTOPLANETARY DISKS
}

\author{
Colin P. McNally ${ }^{1}$, Alexander Hubbard ${ }^{2}$, Chao-Chin Yang ${ }^{3}$, and Mordecai-Mark Mac Low ${ }^{2}$ \\ Draft version June 22, 2021
}

\begin{abstract}
The magnetorotational instability (MRI) drives magnetized turbulence in sufficiently ionized regions of protoplanetary disks, leading to mass accretion. The dissipation of the potential energy associated with this accretion determines the thermal structure of accreting regions. Until recently, the heating from the turbulence has only been treated in an azimuthally averaged sense, neglecting local fluctuations. However, magnetized turbulence dissipates its energy intermittently in current sheet structures. We study this intermittent energy dissipation using high resolution numerical models including a treatment of radiative thermal diffusion in an optically thick regime. Our models predict that these turbulent current sheets drive order-unity temperature variations even where the MRI is damped strongly by Ohmic resistivity. This implies that the current sheet structures where energy dissipation occurs must be well resolved to correctly capture the flow structure in numerical models. Higher resolutions are required to resolve energy dissipation than to resolve the magnetic field strength or accretion stresses. The temperature variations are large enough to have major consequences for mineral formation in disks, including melting chondrules, remelting calcium-aluminum rich inclusions, and annealing silicates; and may drive hysteresis: current sheets in MRI active regions could be significantly more conductive than the remainder of the disk.
\end{abstract}

\section{INTRODUCTION}

In regions of accretion disks where the magnetorotational instability (MRI) acts, differential rotation shears magnetic fields, producing turbulence. The resulting torques extract gravitational potential energy and drive accretion flows (Balbus \& Hawley 1998). In a steady state, the extracted energy must be either exported in a wind, or dissipated locally, heating the disk, which then cools radiatively. In the case of local dissipation, the strength of the accretion flow depends on the nature of the dissipation. The exact nature of the dissipation may also determine the course of mineral formation in protoplanetary disks, as the total amount of energy dissipated suffices to thermally process the solids and ices present (King \& Pringle 2010). The meteoritic record, particularly the chondrites, may reflect these processes.

If that energy dissipation were evenly distributed, it would have little effect on local temperatures. This forms the basis for the common approximation of local isothermality in MRI simulations. However, volume averaged quantities can mislead because the MRI amplified magnetic field, and all its dependent quantities, vary significantly in both time and space. In particular, magnetized turbulence dissipates its energy in current sheets (Parker 1972, 1994, Cowley et al. 1997), quasi-2D structures where the magnetic fields change rapidly in space. This inhomogeneity in space and time is interesting not just because it may control the strength of the accretion flow, but also because it must lead to concentration of

${ }^{1}$ Niels Bohr International Academy, Niels Bohr Institute, Blegdamsvej 17, DK-2100, Copenhagen Ø, Denmark, cmcnally@nbi.dk

2 Department of Astrophysics, American Museum of Natural History, New York, NY 10024-5192, USA

${ }^{3}$ Lund Observatory, Department of Astronomy and Theoretical Physics, Lund University, Box 43, SE-22100, Lund, Sweden energy dissipation, and thus spatial variations in temperature. Indeed, Hirose \& Turner (2011) found that even in regions where stellar irradiation dominates the energy budget, current sheets can locally heat gas to temperatures $50 \%$ greater than that of the gas heated by starlight.

Most simulations of the MRI in protoplanetary disks are at least locally isothermal in design: they do not advance a temperature equation, although the imposed temperature may be a function of radial position. Some of the earliest MRI simulations did include an energy equation, Ohmic heating, thermal diffusion, and a balancing cooling, but these were only low resolution and the Ohmic heating was not resolved (e.g., Brandenburg et al. 1995, 1996).

More recently, some simulations have solved the full radiative transfer problem to determine the temperature structure of a local region of the disk (Turner et al. 2003, Turner 2004, Hirose et al. 2006; Blaes et al. 2007; Krolik et al. 2007; Flaig et al. 2009), and even included stellar irradiation of the disk surface in the case of Hirose \& Turner (2011). A first non-isothermal, global model including radiative transfer was performed by Flock et al. (2013), assuming an initial azimuthal magnetic field geometry. However, none of these models, except those of Hirose \& Turner (2011), included an explicit treatment of heating and magnetic field diffusion caused by resistivity. Hirose \& Turner (2011) included a detailed model of the resistivity in order to study the extent of the MRI-dead zone, but their simulations appear too poorly resolved to capture the full structure of current sheet driven heating. The current sheets they studied form in the upper active layers of a disk section with a midplane dead zone. These current sheets develop where large azimuthally directed flux tubes are driven together. This basic behavior, of the strongest current sheets occurring where oppositely directed azimuthal flux tubes contact, is also found in 
the simulations we describe here.

Fleming et al. (2000) studied net vertical field MRI with finite Ohmic resistivity for a range of resistivities. Two particular qualitative features of those models recur in our study. First, the variation of the Maxwell stress is found to be much greater in MRI with significant Ohmic resistivity than is found at low resistivity, and this variation appears to be connected to quasi-periodic reappearance of MRI channel flows. Second, considerable heating though Ohmic dissipation occurs in their more resistive models. However, their models lack an energy loss mechanism that would allow the system to reach a quasi-steady state at long times. The model we study here includes such a mechanism.

Characterizing the heating effects in current sheets is particularly important because in thermally ionized regions of protoplanetary disks, spatial temperature variation can drive short-circuit instabilities (Hubbard et al. 2012 McNally et al. 2013). This occurs because the ionization fraction, and hence the Ohmic resistivity, is an exquisitely sensitive function of temperature when the temperature is high enough for thermal ionization to set in. If a current sheet forms, Ohmic heating can raise the temperature, increasing the ionization level, which reduces the resistivity, concentrating the current. This leads to even stronger Ohmic heating, causing runaway heating in the sheet.

Even in regions where non-thermal ionization dominates, order unity temperature variations have important effects. In hotter regions, order-unity temperature variations can process rocks, annealing amorphous silicates or melting chondrules. They impact the behavior of the MRI by altering the density and pressure structure of the gas, as well as the strength of ambipolar diffusion (ion-neutral drift) and the Hall effect. Furthermore, even modest temperature variations will transform ice lines into broad regions with thickness a large fraction of their orbital radius, allowing solids and vapor to coexist at the same radial position, with repeated evaporationcondensation cycles (Ros \& Johansen 2013). This could strengthen, compactify, and soften dust grains, allowing for both condensation based growth and enhanced collisional growth.

In this paper we describe local models of MRI including Ohmic resistivity, at sufficiently high resolution to explicitly resolve at least some current sheet structure, and including the full energy equation and an approximate treatment of radiative transport. This allows us to study the spatial and thermal structure of the current sheets. We give an analytic argument that strong temperature fluctuations should be expected in MRI-active regions under some conditions, and indeed find such fluctuations in our models. Further, we found that the resolution requirements to fully capture the dissipation are higher than generally thought, so that many existing studies, such as Hirose \& Turner (2011), appear under-resolved.

The effect of resistivity on the MRI dispersion relation can be parameterized by the Elsasser number

$$
\Lambda \equiv \frac{v_{\mathrm{A}}^{2}}{\eta \Omega_{0}} .
$$

In the literature discussing Ohmic resistivity in the context of the MRI, this quantity has also been termed

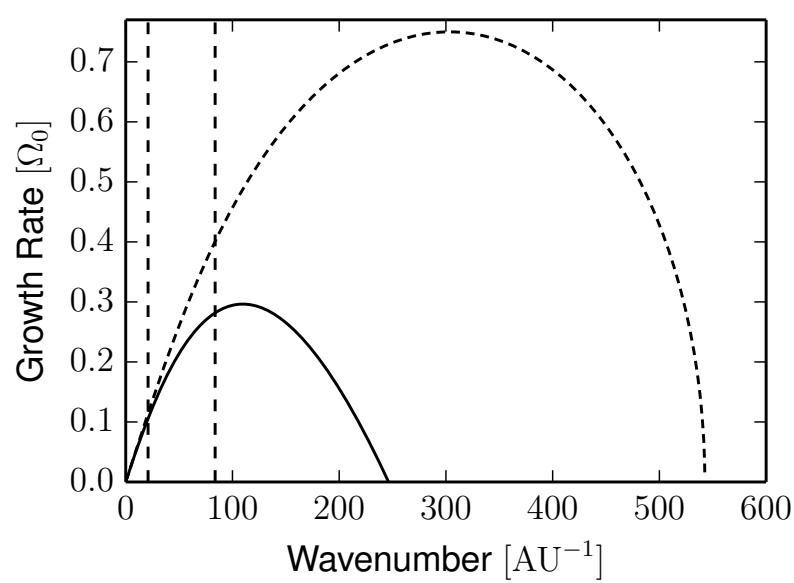

FIG. 1.- Growth rates of zero-resistivity MRI and the specific case of resistive MRI considered here. Dashed: zero resistivity, Solid: Elsasser number $\Lambda=0.5$. Vertical Long Dashed: wavenumbers corresponding to the vertical heights (smallest vertical wavenumber) of the two sizes of shearing box domain of $H$ and $4 H$ used for the simulations in this work.

the magnetic Reynolds number or the Lundquist number. In Figure 1 we show the dispersion relation of the MRI for the parameters studied in this paper (see Table 1) as well as for the ideal case (Jin 1996, Sano \& Miyama 1999, Pessah \& Chan 2008). Considering incompressible axisymmetric perturbations with a net vertical field and Ohmic resistivity, the dispersion relation has two important regimes. For the low resistivity regime, where the Elsasser number is $\Lambda \gg 1$ the most unstable MRI wavelength increases with increasing magnetic field strength and the fastest growing mode growth rate is $\sim 0.75 \Omega_{0}$. However, for the resistive regime with Elsasser numbers $\Lambda<1$, the most unstable MRI wavelength instead decreases with increasing field strength while the fastest growing mode growth rate decreases. The physical regime studied in this paper falls within this second case.

In Section 2 we describe our numerical methods and initial conditions. In Section 3 we present our numerical results on the structure and evolution of current sheets formed in our simulations, and discuss their reliability in Section 4. Section 5 gives an analytic explanation of these results. Section 6 explores how our results depend on the behavior of the opacity and the resistivity, respectively. Finally, in Section 7 we discuss the limitations and implications of our work.

\section{SIMULATIONS}

\subsection{Methods}

We performed simulations of unstratified, magnetized, shearing boxes with net vertical field including Ohmic resistivity and radiative thermal diffusion using the Pencil Code ${ }^{4}$, a sixth-order, central difference in space method, (Brandenburg \& Dobler 2002), as well as confirming our major results with Athena (Stone et al. 2008. Stone \& Gardiner 2010), a constrained-transport, Godunov

4 Details on the Pencil Code and download information can be found at http://www.nordita.org/software/pencil-code/

${ }^{5}$ Details and download information for Athena can be found at https://trac.princeton.edu/Athena/ 
method. Here we use Athena configured with orbital advection, linear reconstruction, and the HLLD Riemann solver.

The field variables evolved in the Pencil Code were density, velocity, magnetic vector potential, and thermal energy density. The Pencil Code requires diffusion operators for stability, so we employed a grid-scaled hyperdiffusion in velocity and density (which have no physically resolved diffusion effect in the model), along with shock diffusion on all fields so that all shocks are captured at the grid scale. Because the Pencil Code is sixth order accurate in space and third order accurate in time, it resolves small scale subsonic flow structures almost as well as a spectral code. However, it does not conserve energy exactly. To confirm our results, we replicated the configuration in Athena, a locally energy-conservative, finite volume code. In such a method, no explicit stabilizing diffusion on the momentum and density fields is required. Furthermore, the conversion of energy from kinetic and magnetic energy in shocks and current sheets to thermal energy is fully conservative.

We included two explicit physical diffusive effects in our model, resistivity $\eta$, and radiative thermal diffusion. We allowed neither $\eta$ nor the opacity $\kappa$ to vary with temperature, unlike in an actual disk. The form of our Ohmic resistivity is

$$
\eta \equiv \frac{c^{2}}{4 \pi \sigma}
$$

in Gaussian cgs units, where $\sigma$ is the conductivity. The evolution of the magnetic field $\boldsymbol{B}$ is then described by the induction equation

$$
\frac{\partial \boldsymbol{B}}{\partial t}=\nabla \times\left(\left[\boldsymbol{v}-\frac{3}{2} \Omega_{0} x \hat{\boldsymbol{y}}\right] \times \boldsymbol{B}\right)-\frac{4 \pi}{c} \nabla \times[\eta \boldsymbol{J}],
$$

where the current

$$
\boldsymbol{J} \equiv \frac{c}{4 \pi} \nabla \times \boldsymbol{B},
$$

and the shearing box fluctuation velocity is $\boldsymbol{v}$. The $(3 / 2) \Omega_{0} x \hat{\boldsymbol{y}}$ term is the orbital shear.

So long as the resistivity remains constant in space,

$$
\frac{\partial \boldsymbol{B}}{\partial t}=\nabla \times\left(\left[\boldsymbol{v}-\frac{3}{2} \Omega_{0} x \hat{\boldsymbol{y}}\right] \times \boldsymbol{B}\right)-\eta \nabla \times[\nabla \times \boldsymbol{B}] .
$$

The resistive term was integrated with the same scheme as the other operators. The Pencil Code evolves an analogous equation for the magnetic vector potential $\boldsymbol{B}=\nabla \times \boldsymbol{A}$.

The thermal energy evolution includes a diffusive term based on radiative transfer in the optically thick limit with Rosseland mean opacities, as well as a thermal relaxation term to model the large scale energy transfer away from the midplane of the disk. The full thermal energy equation is thus

$$
\begin{aligned}
\frac{\partial e}{\partial t}=- & \nabla\left(\left[\boldsymbol{v}-\frac{3}{2} \Omega_{0} x \hat{\boldsymbol{y}}\right] e\right)-p_{\mathrm{th}} \nabla \cdot \boldsymbol{v} \\
& +\frac{4 \pi \eta}{c^{2}} J^{2}-\nabla \cdot \boldsymbol{F}-C_{v} \frac{T-T_{0}}{\tau_{0}}
\end{aligned}
$$

where $p_{\mathrm{th}}=(\gamma-1) e=\rho k_{B} T / \bar{m}$ is the thermal pressure, $C_{v}=(\gamma-1)^{-1} k_{B} / \bar{m}$ the heat capacity at constant volume, $T$ is the gas temperature, $T_{0}$ the reference temper-
TABLE 1

Parameter Values

\begin{tabular}{lll}
\hline & Parameter & Value \\
\hline$\rho_{0}$ & Initial density & $10^{-9} \mathrm{~g} \mathrm{~cm}^{-3}$ \\
$T_{0}$ & Background temperature & $950 \mathrm{~K}$ \\
$L_{x}$ & Box size in $x$ & $0.3 \mathrm{AU}$ \\
& & $4.85 \mathrm{H}$ \\
$\Omega_{0}$ & Orbital frequency & $2 \pi \mathrm{yr}^{-1}$ \\
$r_{0}$ & Shearing box position & $1 \mathrm{AU}$ \\
$\gamma$ & Gas adiabatic Index & 1.5 \\
$\bar{m}$ & Gas mean particle mass & $2.33 \mathrm{amu}$ \\
$\eta$ & Ohmic resistivity $c^{2} / 4 \pi \sigma$ & $8.9 \times 10^{14} \mathrm{~cm}^{2} \mathrm{~s}^{-1}$ \\
& & $5.2 \times 10^{-3} \Omega H^{2}$ \\
$\beta_{0}$ & Initial plasma beta & 750 \\
$v_{A 0}$ & Initial Alfvén speed & $9.5 \times 10^{3} \mathrm{~cm} \mathrm{~s}^{-1}$ \\
& & $5.2 \times 10^{-2} \Omega \mathrm{H}^{-1}$ \\
$\Lambda_{0}$ & Initial Elsasser number & 0.5 \\
$\kappa$ & Rosseland mean opacity & $20 \mathrm{~cm}^{2} \mathrm{~g}^{-1}$ \\
$\tau_{0}$ & Thermal relaxation time & $1 \mathrm{yr}$ \\
$\lambda_{\mathrm{MRI}}$ & MRI fastest growing mode & $5.7 \times 10^{-2} \mathrm{AU}$ \\
& & $0.92 \mathrm{H}$
\end{tabular}

ature, $\tau_{0}$ the thermal relaxation time, $k_{B}$ the Boltzmann constant, $\bar{m}$ the mean mass per particle, and with the Rosseland mean opacity thermal diffusion flux $\boldsymbol{F}$ given by

$$
\boldsymbol{F}=-\frac{16 \sigma_{B} T^{3}}{3 \rho \kappa} \nabla T
$$

where $\sigma_{B}$ is the Stefan-Boltzmann constant, $\rho$ the density and $\kappa$ the Rosseland mean opacity.

The thermal diffusion treatment is motivated by the high optical depth expected over length scales of the MRI unstable wavelength expected for the outer edge of the MRI active region in the dusty inner disk. In this region $\lambda_{\mathrm{MRI}} \simeq H$, so the optical depth of an MRI wavelength is comparable to that of the disk itself. For our parameters, $\lambda_{\text {MRI }}$ has an optical depth $\tau>15,000$. We choose the thermal relaxation timescale to be an orbital period $\tau_{0}=2 \pi / \Omega_{0}$ (Table 1 ). We make this choice to probe the coolest reasonable disk state, which corresponds to one that can cool so quickly that it approaches marginal gravitational stability. The thermal relaxation rate is chosen to be fast, so that it strongly limits the heating resulting from the energy input by the background shear. Additionally, we can place an upper limit on the physically plausible cooling rate by recognizing that the model is aimed to produce the environment at the inner edge of a dead zone. If the cooling rate of the neighboring dead zone was much faster than $\tau_{0}=\Omega_{0}^{-1}$, the region would become gravitationally unstable (Rice et al. 2014), and would thus be prone to generating strong spiral waves, strongly altering the global disk structure. As such, we can argue that this region of the disk must, to be consistent with an MRI-unstable quasi-steady state at the inner edge of the dead zone, have a cooling timescale longer than $\tau_{0}=\Omega_{0}^{-1}$. Therefore, our choice of $\tau_{0}=\Omega_{0}^{-1}$ should be a conservative one, and should result in a model which underestimates the temperature fluctuations.

\subsection{Initial Conditions}

We chose the parameters listed in Table 1 to approximate a disk that is marginally MRI active at 1 AU. Con- 
ceptually, these parameters can be thought of as corresponding to a region at the inner edge of a dead zone. Unusually for astrophysical magnetohydrodynamics, effects of the microphysical resistivity are resolvable at the inner edge of the dead zone (unlike the unresolvable microphysical viscosity) because there the MRI is marginally super-critical and numerical simulations can clearly show the growth of magnetic fields in an MRI dynamo.

The marginal instability criterion for the MRI is that the most unstable wavelength of the MRI be comparable to the disk scale height. In our case, the MRI wavelength $\lambda_{\text {MRI }}$ and the effective scale height of the disk $H \equiv c_{s} / \sqrt{\gamma} \Omega$ are within $10 \%$ of each other. This means that even though we have performed unstratified numerical simulations, we can link our length scales to those of stratified simulations near MRI-criticality. With these parameters, the initial net vertical magnetic field corresponds to an initial plasma $\beta_{0}=750$. This is low compared to many existing models with zero resistivity. However, in this very resistive regime the wavelength of the fastest growing MRI mode increases with $\beta_{0}$. Accordingly, a relatively strong initial field is needed to make the most unstable length scale match the nominal disk scale height. The wavenumbers corresponding to the vertical heights of the shearing box domains used in this study are noted in Figure 1 along with the dispersion relation curve for the MRI.

While temperature is not strictly diffused, for the parameters in Table 1, extrapolating from Equation (7) and using $T=(\gamma-1) \bar{m} e / \rho k_{B}$, an effective thermal temperature diffusion coefficient can be estimated as

$$
\mu \equiv \frac{16(\gamma-1) \bar{m} \sigma_{B} T_{0}^{3}}{3 \rho_{0}^{2} \kappa k_{B}} \simeq 1.8 \times 10^{14} \frac{\mathrm{cm}^{2}}{\mathrm{~s}},
$$

where $\sigma_{B}$ is the Stefan-Boltzmann constant, and the other parameters are as defined in Table 1. When measured with respect to the MRI wavelength and orbital timescale of our setup, we have

$$
\mu \sim \frac{\lambda_{\mathrm{MRI}}^{2} \Omega_{0}}{1000}
$$

Accordingly, the radiative cooling time for a structure with the size of the most unstable MRI wavelength is approximately $130 \mathrm{yr}$, far longer than the imposed cooling time of $1 \mathrm{yr}$. This means that large scale thermal structures are dissipated by the thermal relaxation cooling term, but small scale structures (less than $0.1 \lambda_{\mathrm{MRI}}$ ) cool radiatively.

\subsection{Runs}

We used the Pencil Code to perform two sequences of runs. The first sequence uses a cubic volume with res-

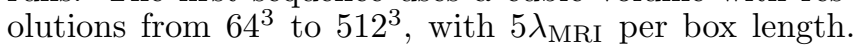
Our highest two resolutions are equivalent to 50 and 100 zones per scale height, respectively. The higher resolution runs were initialized from the next highest resolution run. The $64^{3}$ simulation was run for $t=105$ orbits. At $t=45$ orbits, it was remeshed to a resolution of $128^{3}$ and this was also run to $t=105$ orbits. At $t=60$ orbits, the $128^{3}$ run was remeshed to $256^{3}$ and this was run to $t=105$ orbits. Finally, at $t=75$ orbits, the $256^{3}$ run was remeshed to $512^{3}$ and this was run to $t=105$ orbits. The second sequence of runs repeats the same parame- ters with a slab volume one quarter the height, that is with aspect ratio $L_{x}: L_{y}: L_{z}$ of $1: 1:(1 / 4)$. Thus, these runs use a shearing box with a height of approximately one scale height. These runs preserved the same cell-size as the first sequence of runs, so the highest resolution was $512^{2} \times 128$. Otherwise the procedures and analysis of this sequence of runs follows the cubical runs.

The comparison runs done with Athena used the cubical grid and did not use remeshing. Both started from $t=0$ and ran for 50 orbits. Resolutions of $128^{3}$ and $256^{3}$ are reported here. The setup is otherwise identical to that used with the Pencil Code.

\section{RESULTS}

\subsection{Global Averages}

We first consider the behavior of the global average quantities in our models. In the top panels of Figures 2 and 3 we show time series of the volume average plasma beta

$$
\bar{\beta}=\frac{8 \pi\left\langle p_{\mathrm{th}}\right\rangle_{V}}{\left\langle B^{2}\right\rangle_{V}}
$$

where $\langle\cdots\rangle_{V}$ denotes volume averaging, for our different resolution runs. The volume average of the magnetic field was taken separately to avoid having this average skewed by very high values of $\beta$ within demagnetized reconnecting regions. Our cubical models settle at values of $\bar{\beta} \sim 10$, while the slab models only reach $\bar{\beta} \sim 50$. Similarly, the bottom panel of both figures shows the volume averaged Shakura \& Sunyaev (1973) $\alpha$ turbulent viscosity parameter:

$$
\alpha=\frac{1}{p_{\mathrm{th}}}\left(\rho v_{x} v_{y}-\frac{B_{x} B_{y}}{4 \pi}\right) .
$$

The volume averaged value of this parameter settles at a value of about $\langle\alpha\rangle_{V}=4 \times 10^{-2}$ in the cubical case and $\langle\alpha\rangle_{V}=3 \times 10^{-2}$ in the slab case.

In Figures 4 and 5 we show temperature and heating data from the Pencil Code runs. Two facts jump out. First, there is an order unity difference between the tenth and ninetieth percentile temperatures at all times. Second, the dominant energy dissipation mechanism is resistive rather than compressive or shock dissipation, because when averaged over the volume, the adiabatic heating and cooling approximately cancel. This occurs even though the compressive heating has much stronger peak values than the resistive heating, as we show in the next section.

\subsection{Two-dimensional Slices}

To more closely investigate the source of the temperature variations, we turn to a study of the morphology of current sheets. We find that the heating is usually dominated by one or two major sheets at any given time. In Figure 6 we show a set of slices at constant azimuth through our highest resolution cubical Pencil Code run. In the slice chosen, there is a current sheet lying in the radial-azimuthal plane, perpendicular to the slice plane, and visible in all variables except the total pressure, although only barely visible in the adiabatic heating and cooling panel. This figure makes clear that, even though the volume average $\bar{\beta} \simeq 8$ at the orbit in question, the 

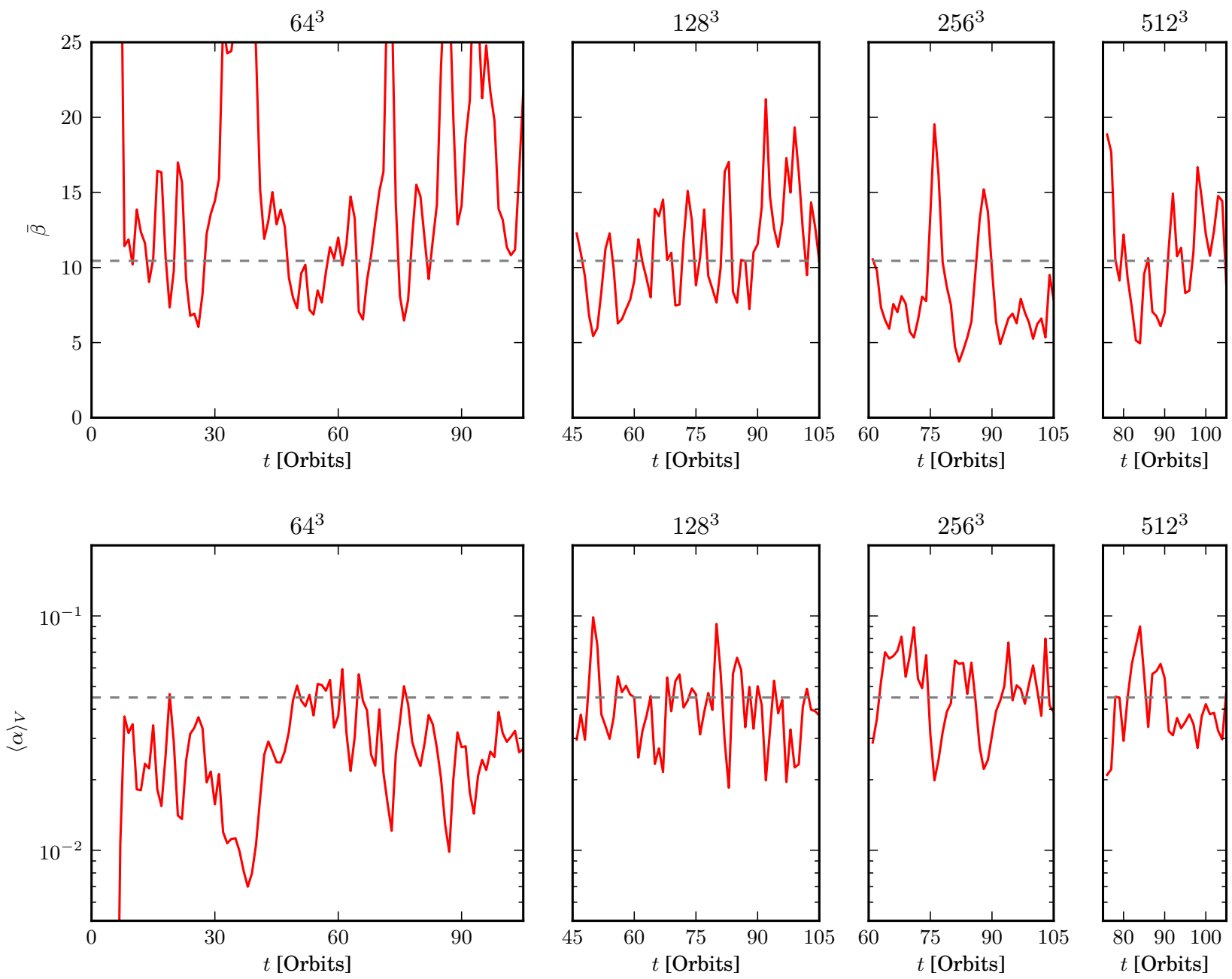

FIG. 2.- Volume averages of the plasma $\bar{\beta}$ and total stress $\langle\alpha\rangle_{V}$ for the cubical Pencil Code runs. Note that the scale of the time-axis is the same for all panels, but the higher resolution runs have shorter total run-time, as they were remeshed from the next highest resolution run. The average value for the highest resolution runs are shown by the dashed lines to guide the eye.

local minimum ratio associated with the peak magnetic field is $\beta_{p} \simeq 1$.

Furthermore, the largest temperature variation generally traces the highest resistive dissipation in the current sheet structure, although there is enough difference between the two to make clear that it is time-averaged, rather than instantaneous, heating that determines the temperature perturbation. Although the compressive heating is high along the weak shocks filling the domain, these are accompanied by large regions of expansion cooling. Hence, as was seen in the volume average shown in the upper panel of Figure 4 , the heating is dominated by the resistive dissipation.

Similarly, Figure 7 shows a set of slices at constant azimuth through our highest resolution slab run. The qualitative pattern of dominant azimuthal field bundles is similar, and a strong, hot current sheet structure can be seen. Consistent with the results for the globally averaged quantities, the values are less extreme in this vertically restricted domain than in the cubic one: the volume averaged $\bar{\beta} \simeq 20$ and the local minimum $\beta$ associated with the peak magnetic field is $\beta_{p} \simeq 1$.

This difference between the local $\beta_{p}$ and volume aver- aged $\bar{\beta}$ is straightforward. The strongest magnetic field structures of the MRI are generated by orbital shear, which stretches radial magnetic field lines into nearly azimuthally constant bundles of strong, azimuthally directed magnetic flux. If the magnetic field is dominantly contained in azimuthally constant structures varying along a single large scale wave-vector $\boldsymbol{k}$, we would expect $\beta_{p} \simeq \bar{\beta} / 4$ because we need to average over $\sin \left(k_{x} x\right)^{2} \sin \left(k_{z} z\right)^{2}$. If $\beta_{p}$ is of order unity, its value is expected to further drop because the magnetic field bundles will have low thermal pressure compared to the volume averaged thermal pressure (because the magnetic pressure pushes fluid out of the high magnetic field regions). In Figure 8 we show a vertical cut through the current sheet identified in Figure 6 that demonstrates that this inverse magnetic and thermal pressure correlation occurs in large current sheets found in the highest resolution aspect ratio cubical model.

These figures also show that even though the simulation has $\beta_{p} \sim 1$ and hence drives mildly supersonic flows, the large temperature variations are due to resistive heating. Regions heated by the hydrodynamical 

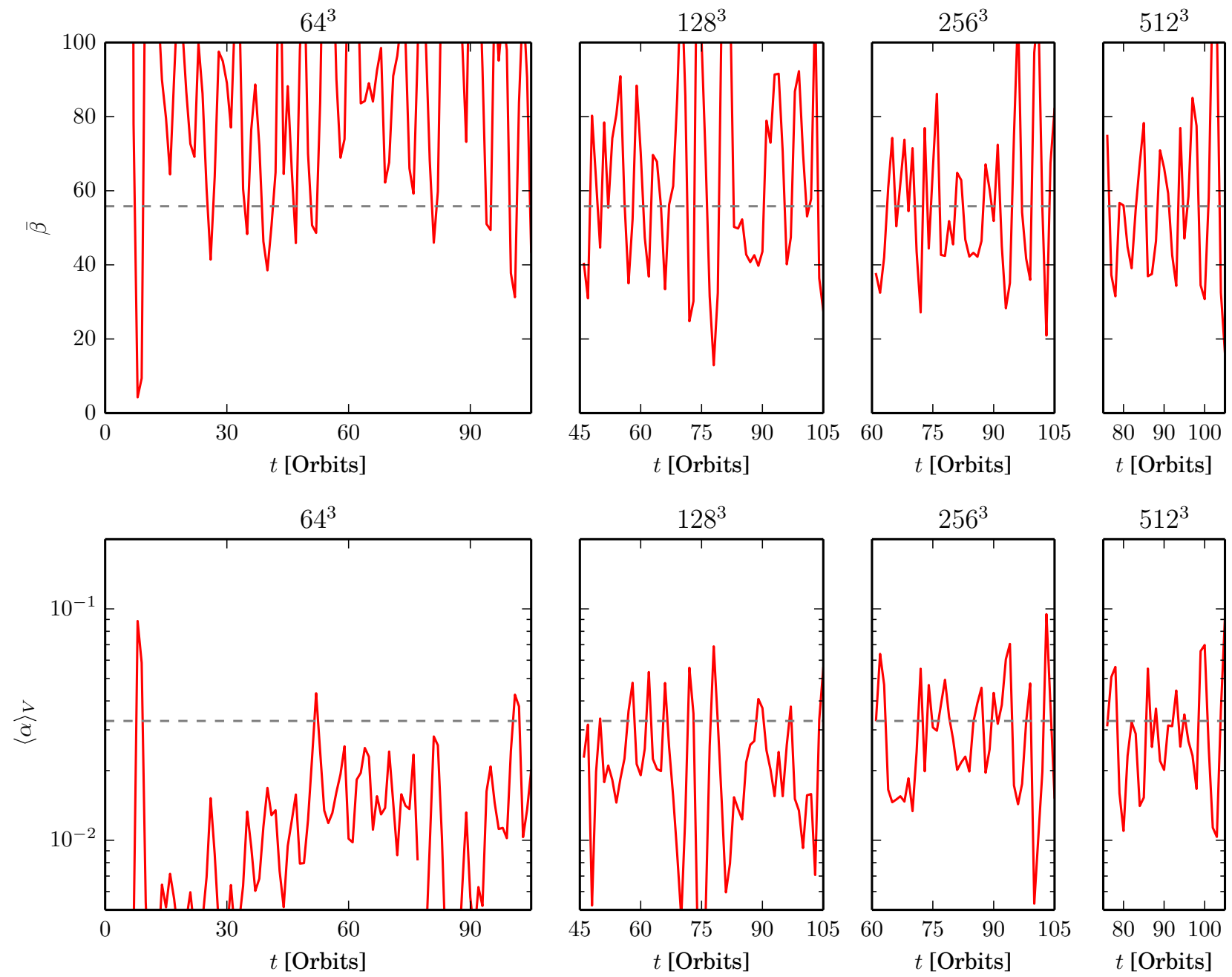

FIG. 3.- Volume averages of the plasma $\bar{\beta}$ and total stress $\langle\alpha\rangle_{V}$ for the slab Pencil Code runs. Note that the scale of the time-axis is the same for all panels, but the higher resolution runs have shorter total run-time, as they were remeshed from the next highest resolution run. The average value for the highest resolution runs are shown by the dashed lines to guide the eye.

shocks quickly reexpand and adiabatically cool, leaving little imprint on the temperature structure of the gas.

\section{MODEL RELIABILITY}

Our conclusions depend on resolving the heating within thin current sheets. We therefore have made a detailed study of the convergence properties of our models, and confirmed our Pencil Code results by comparison to models run with an independent numerical method.

\subsection{Global Averages}

Figures 2 and 3 compare the time variation of $\bar{\beta},\langle\alpha\rangle_{V}$, in runs of varying resolution for the two aspect ratios. The average value for the highest resolution runs are shown by the dashed lines to guide the eye. Similarly Figures 4 and 5 compare the temperature and heating rates. Episodes of very low field strength are common for the lower resolution runs, which are less well resolved, and hence subject to stronger numerical resistivity. Our simulations appear to have converged to at least the intrinsic time variation in these averaged quantities for resolutions of $128^{3}$ or larger. However, the temperature and the resistive heating rate converge only for the two high- est resolutions $\left(256^{3}\right.$ and $512^{3}$, or 50 and 100 zones per $\lambda_{\mathrm{MRI}}$ or equivalently per scale height.).

The motivation for the thinner domain is to avoid the cycles of strong channel flow and intermittency that have been observed to be exacerbated by the use of a cubic domain (Bodo et al. 2008). Indeed, the variation on $\sim 10$ orbit timescale appears less in these runs than in the cubical ones. However, channel mode-like pairs of azimuthal field bundles still dominate the magnetic field, as can be seen in the top left panel of Figure 7. The heating is less extreme for the thinner box, but the largest MRI active scales are also truncated by the vertical height (see Figure 11. Accordingly, the volume average $\bar{\beta}$ is on the order of five times higher in these runs.

We explain the increase in volume averaged heating rate with resolution by reference to the theory described in Section 5.2. Magnetic fields are current sources: $\boldsymbol{J} \propto \boldsymbol{\nabla} \times \boldsymbol{B}$. The MRI often produces oppositely directed azimuthal magnetic field bundles. Given two such bundles with strength $B_{0} / 2$ separated by a distance $L$, the current flowing between them scales as $J \sim B_{0} / L$. However, resistive dissipation scales with the square of the current, $\eta|\boldsymbol{J}|^{2}$, so the net resistive heating scales as 

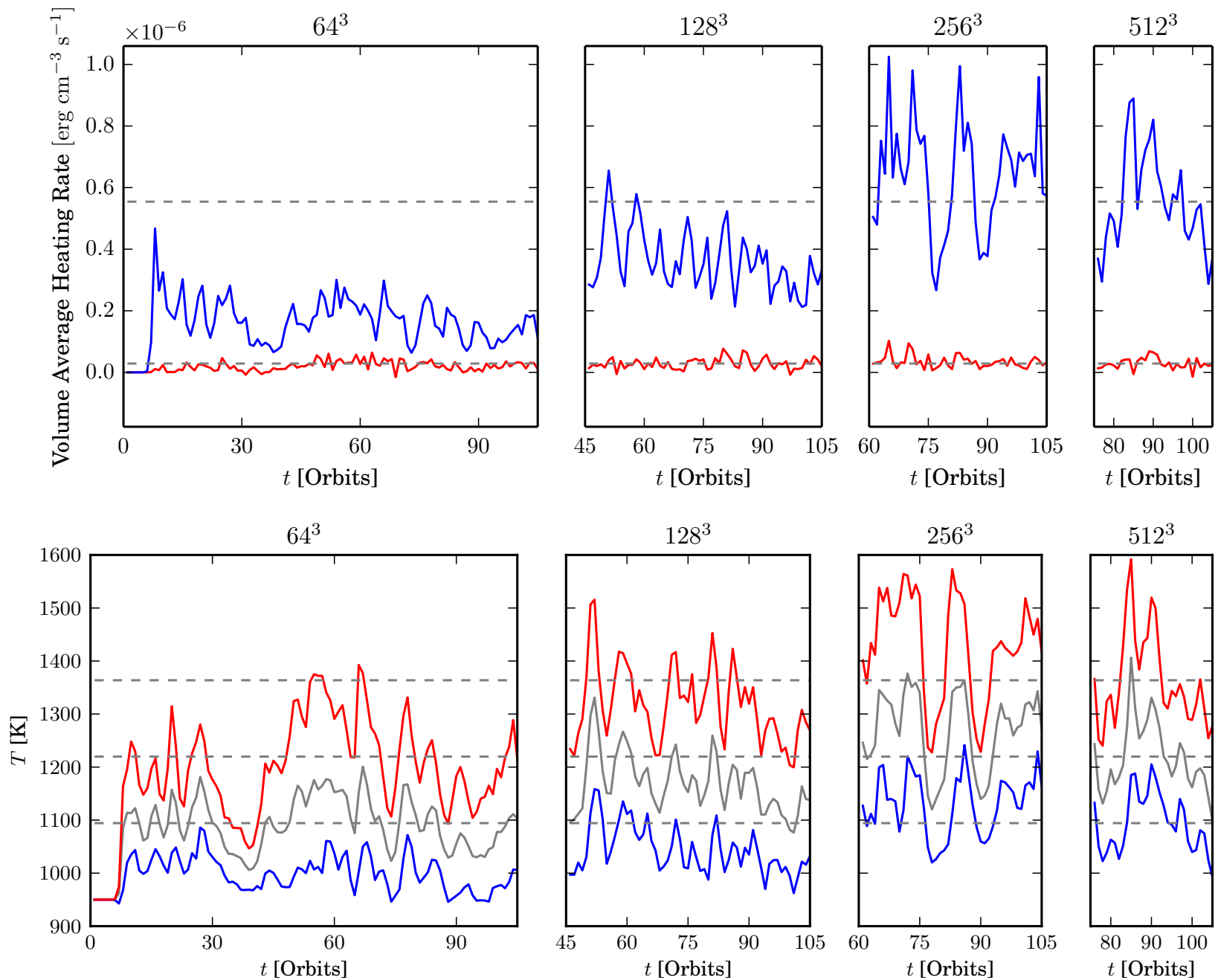

FIG. 4.- Upper panel: volume averaged heating rate for the cubical Pencil code runs. Resistive heating in blue and compressive heating or cooling in red. Lower panel: ninetieth percentile (red), fiftieth percentile (gray) and tenth percentile (blue) temperatures for the same runs. Higher resolution runs have shorter run times as they were remeshed from the next highest resolution run. For each data series, the average value for the highest resolution runs are shown by the dashed lines to guide the eye.

$\eta B_{0}^{2} / L^{2}$. If the current sheet is not resolved the heating rate will be understated because the actual value of $L$, set by the resolution, is larger than the physical one set by $\eta$.

The high resolution required to resolve the current sheets, even at the high resistivity edge of the dead zone, can be understood from dimensional considerations. $\mathrm{Nu}-$ merical resistivity $\eta_{n} \propto u \times \Delta x$, where $u$ is the turbulent velocity. If the imposed resistivity

$$
\eta \gg \eta_{n}
$$

then the physical resistivity $\eta$ dominates over the numerical resistivity $\eta_{n}$ in Equation (3) and resistive effects are resolved.

Assuming equipartition between the turbulent kinetic and turbulent magnetic energies, $u^{2} \sim v_{A}^{2}$, so $\eta_{n} \sim$ $v_{A} \times \Delta x$, where $v_{A}$ is the Alfvén speed of the saturated magnetic field. The ratio of $\eta_{n}$ for the saturated state to $\eta_{n 0}$ of the initial state is $\eta_{n} / \eta_{n 0} \simeq v_{A} / v_{A 0}=\left(\beta_{i} / \beta_{s}\right)^{1 / 2}$ where $\beta_{i}$ is the plasma beta of the initial field, and $\beta_{s}$ is that of the saturated field. This ratio is often an order of magnitude or larger.

This can cause issues even near the relatively high resistivity edge of the dead zone where Equation $\sqrt{12}$ is best satisfied. The MRI criticality condition is that the initial Elsasser number $\Lambda_{0}=v_{A 0}^{2} / \eta \Omega$ be of order unity, where $v_{A 0}$ is the Alfven speed of the seed field. The magnetic Reynolds number of the saturated state is

$$
\operatorname{Re}_{M} \equiv u^{2} t_{t} / \eta
$$

where $t_{t}$ is the turbulent turnover time, $t_{t} \simeq \Omega^{-1}$, and taking $u$ to be the maximum shearing-box fluctuation velocity. In our case, the initial Elsasser number is $\Lambda_{0}=0.5$ and at late times the turbulent magnetic Reynolds number is $\operatorname{Re}_{M} \sim 100$, which is a non-trivial value of $\operatorname{Re}_{M}$ to resolve numerically. Note that $\operatorname{Re}_{M} / \Lambda_{0}=\left(\eta_{n} / \eta_{n 0}\right)^{2}$, so the grid resolution needed to resolve the turbulent flow in the saturated state is a factor of over 14 times more stringent than at the initial time. All our runs, including the lowest resolution, $64^{3}$ ones, resolved the initial state according to Equation (12). During the highest energy episodes, however, even the highest resolution $512^{3}$ 

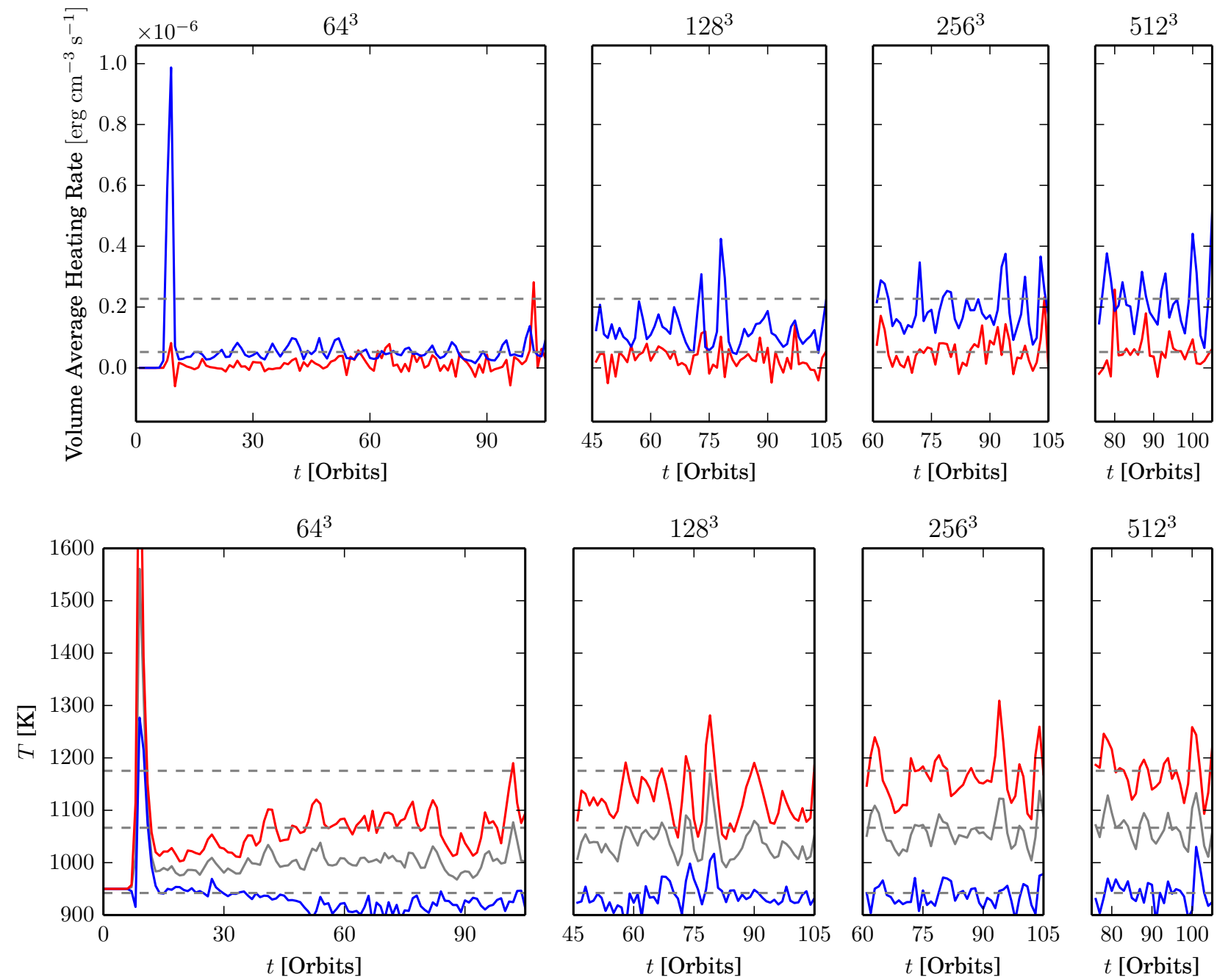

FIG. 5.- Upper panel: volume averaged heating rate for the slab Pencil code runs. Resistive heating in blue and compressive heating or cooling in red. Lower panel: ninetieth percentile (red), fiftieth percentile (gray) and tenth percentile (blue) temperatures for the same runs. Higher resolution runs have shorter run times as they were remeshed from the next highest resolution run. For each data series, the average value for the highest resolution runs are shown by the dashed lines to guide the eye.

simulations did not quite satisfy Equation $(12)$, though only by a factor of about two. To understand the actual convergence behavior, we need to move beyond that criterion's simple dimensional analysis.

\subsection{Decomposition of $|\boldsymbol{J}|^{2}$}

To better probe the resolution required to resolve resistive dissipation we examine the structure of the current density. Our current sheets show multiple lengthscales: the full box length in azimuth, $\lambda_{\mathrm{MRI}}$ in width and a far shorter length perpendicular to the sheet. This rules out a simple Fourier analysis of the current sheet structures. Instead, to examine the convergence of the complex current sheet structures we decomposed $|\boldsymbol{J}|^{2}$ with a multiresolution decomposition (Harten 1994).

We successively coarse-grained the data, and subtracted the coarse-grained data from the original. We call the results of the subtraction residuals, although they are described as error coefficients in other contexts. They measure how much structure there is on a given scale and hence at what scale the dominant features of $|\boldsymbol{J}|^{2}$ exist. The decomposition is conservative, in that the sum of the residuals on every level over the entire volume is zero. To detect how much structure in the field resides on each level, we examine the average absolute value of the residuals on each grid level.

The transform used to calculate the residuals is formally

$$
v^{L} \longleftrightarrow\left\{e^{L}, \ldots, e^{1}, v^{0}\right\}
$$

The function $v$ is on the finest mesh $v^{L}$, the residuals on each level $N$ are $e^{N}$, and $v^{0}$ is the representation of the function $v$ on the coarsest mesh: a single point. The transform uses a decimation operator to move the representation of the function $v$ from mesh level $N$ to a mesh with half as many points in each direction on level $N-1$ by

$$
v_{i, j, k}^{N-1}=\frac{1}{8} \sum_{l, m, n=0}^{1} v_{i+l, j+m, k+n}^{N}
$$

which is a simple average over a cube of eight neighboring points. In this way the volume integral of $v$ is conserved in the successively coarser representations. Thus 

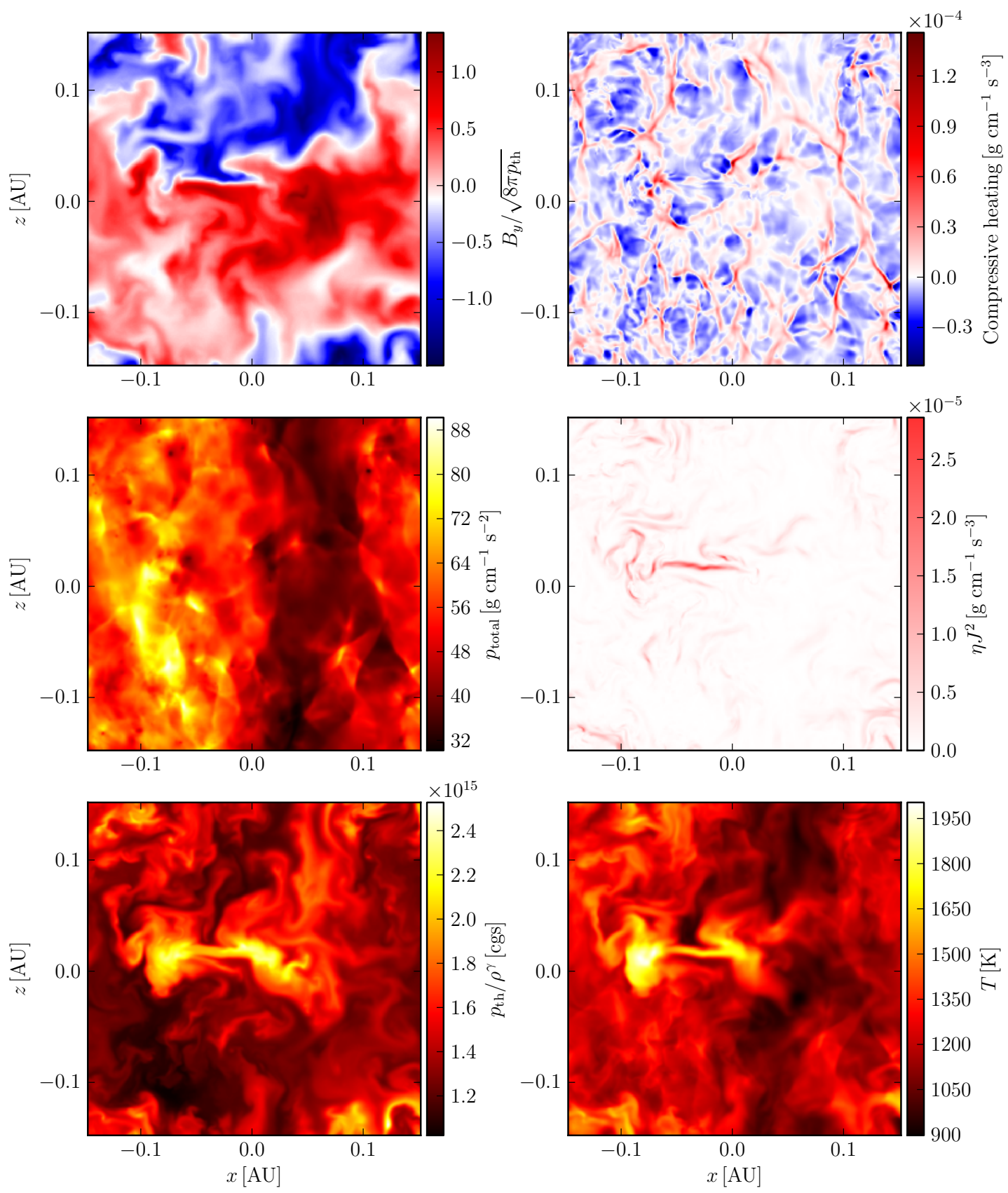

FIG. 6. - Data from a radial-vertical slice of the cubical, $512^{3}$ Pencil Code run at time $t=83$ orbits and azimuth $y=0$ AU, cutting through the largest current sheet on the grid. Top left panel: azimuthal magnetic field, showing a large scale vertical dependence. Middle left panel: total pressure, showing a large scale horizontal dependence known as a zonal flow. Bottom left panel: entropy, showing a maximum in the current sheet centered near $(-0.03 \mathrm{AU}, 0.015 \mathrm{AU})$, so the temperature of the current sheet is not primarily due to adiabatic compression. Top right panel: compressive heating and cooling, showing that shocks permeate the domain, but the current sheet does not stand out. Middle right panel: Resistive heating, clearly showing the current sheet, using the same color scale as the top right panel. Bottom right panel: temperature, showing that the temperature near the current sheet far exceeds the background.

the most coarse representation $v^{0}$ is a scalar value which is equal to the volume average of $v^{L}$. The residuals on each level are defined by the difference between the representation of the function $v$ on level $N$ and level $N-1$ as

$$
\begin{aligned}
e_{2 i+l, 2 j+m, 2 k+n}^{N}= & v_{2 i+l, 2 j+m, 2 k+n}^{N}-v_{i, j, k}^{N-1} \\
& l, n, m \in\{0,1\} .
\end{aligned}
$$

The mean absolute value of the residual $e^{N}$ on level $N$, denoted as $\left\langle\left|e^{N}\right|\right\rangle$ is then a measure of the total volumeintegrated changes between $v^{N-1}$ and $v^{N}$.

If we examine the set of values $\left\{\left\langle\left|e^{1}\right|\right\rangle, \ldots,\left\langle\left|e^{L}\right|\right\rangle\right\}$, the location of the largest residual corresponds to the grid scale where the original function $v^{L}$ changes most. The single $\left|v^{0}\right|$ value measures the volume averaged $|\boldsymbol{J}|^{2}$. To 

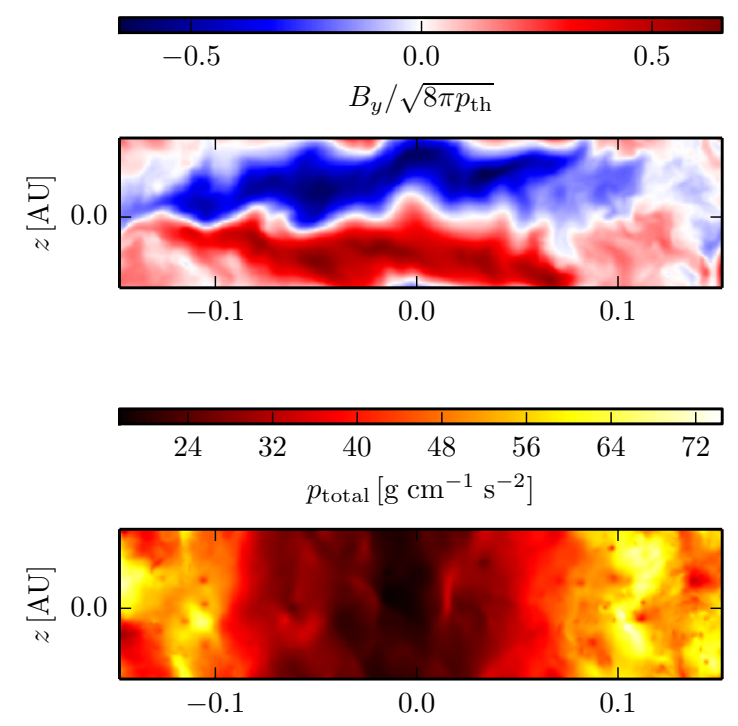

$\begin{array}{lllllllll}1.04 & 1.12 & 1.20 & 1.28 & 1.36 & 1.44 & 1.52 & 1.60 & 1.68\end{array}$ $p_{\mathrm{th}} / \rho^{\gamma}[\mathrm{cgs}]$ $\times 10^{15}$

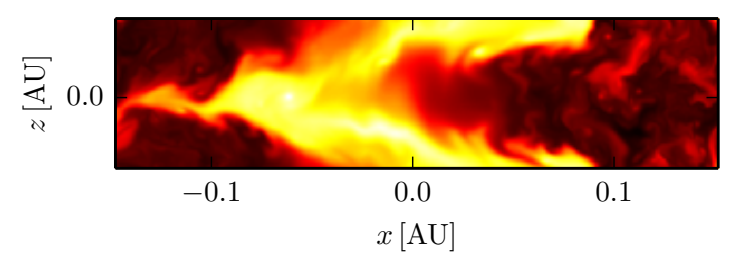

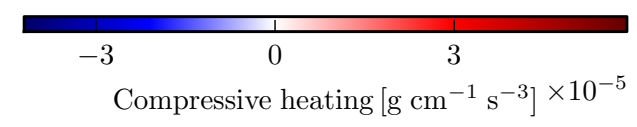
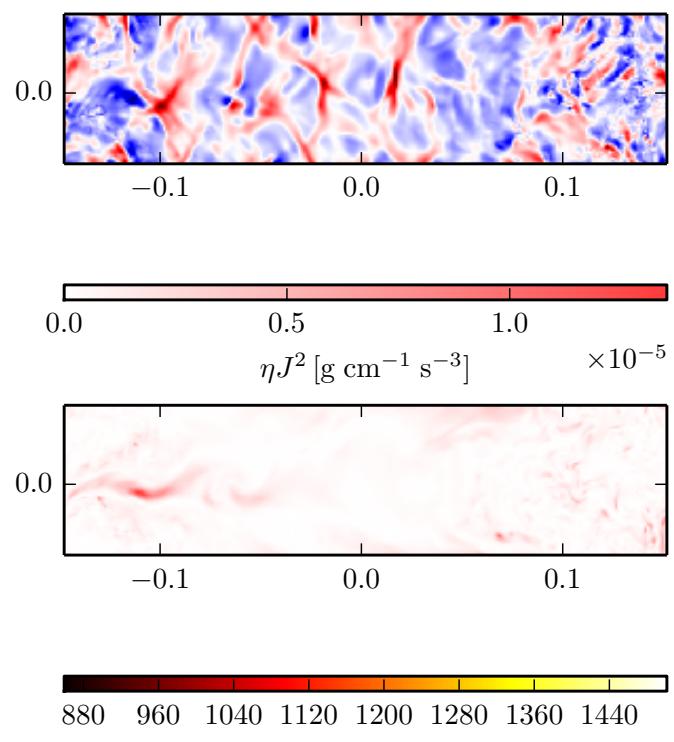

$T[\mathrm{~K}]$

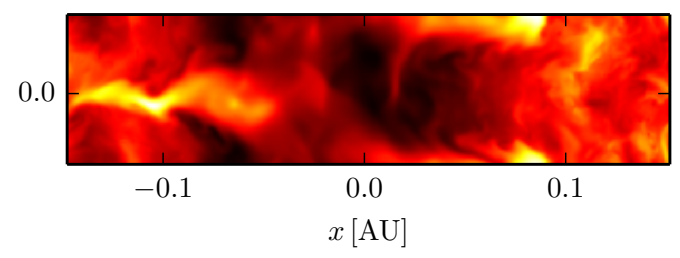

FIG. 7. - Data from a radial-vertical slice of the $512^{2} \times 128$ slab Pencil Code run at time $t=105$ orbits and azimuth $y=0.15$ AU, cutting through the largest current sheet on the grid. Top left panel: Azimuthal magnetic field, showing a large scale vertical dependence. Middle left panel: Total pressure, showing a large scale horizontal dependence known as a zonal flow. Bottom left panel: Entropy, showing a maximum in the current sheet centered near $(-0.1 \mathrm{AU}, 0.0 \mathrm{AU})$, so the temperature of the current sheet is not primarily due to adiabatic compression. Top right panel: Compressive heating and cooling, showing that shocks permeate the domain, but the current sheet does not stand out. Middle right panel: Resistive heating, clearly showing the current sheet, using the same color scale as the top right panel. Bottom right panel: Temperature, showing that the temperature near the current sheet far exceeds the background.

account for the temporal variations of the system, we compute this decomposition once per orbit, and report averages of the absolute values of the coefficients, normalized to the time average of $\left|v^{0}\right|$ from the highest resolution simulation.

The results of this analysis are shown in Figures 9 and 10 . The time averages were performed over the interval $t=80$ to $t=105$. The $\left|v^{0}\right|$ values show that the volume integrated $|\boldsymbol{J}|^{2}$ only approaches convergence at resolution of $256^{3}$ to $512^{3}$. Both the cubical and slab simulations show the same convergence behavior. The averaged residuals $e^{N}$ consistently peak at the scale corresponding to level 5; as resolution increases, the finest scales show decreasing $e^{N}$ coefficients. The strength of the residuals converges well at the highest resolutions. Note that $e^{5}$ corresponds to the structure of $\mathrm{J}^{2}$ on a mesh with resolution $32^{3}$, i.e. to a scale of approximately $\lambda_{\mathrm{MRI}} / 6$. Hence, even though $|\boldsymbol{J}|^{2}$ shows structure on all scales, our highest resolution simulations are well able to resolve the dominant scales of current sheet dissipation.

\subsection{Athena Comparison}

To verify that the results from the Pencil Code resolution study are not dominated by any possible local, nonconservative, energy dissipation, we have performed a smaller set of runs with Athena, which conserves energy to numerical accuracy. These runs all start from $t=0$ and are run to $t=50$ orbits. As expected, they display a different convergence behavior and should be compared to the highest resolution Pencil Code result. The volume average $\bar{\beta}$ at $128^{3}$ and $256^{3}$ shown in Figure 11 gives reasonable agreement with the Pencil Code result, generally varying in the range $\bar{\beta}=5-10$. The $\langle\alpha\rangle_{\mathrm{V}}$ viscosity also agrees reasonably, varying in the range $0.05-0.1$, as shown Figure 12. Finally, the variation in temperatures seen in Athena in Figure 13 also agrees reasonably, with the mean temperature typically $\sim 1200 \mathrm{~K}$ and the spread between the $10 \%$ and $90 \%$ percentile values being typically $\sim 200 \mathrm{~K}$. As the Godunov method employed in Athena does not capture shocks through an explicit shock viscosity, it is difficult to decompose the heating rates as was done for the Pencil Code runs. Altogether, 

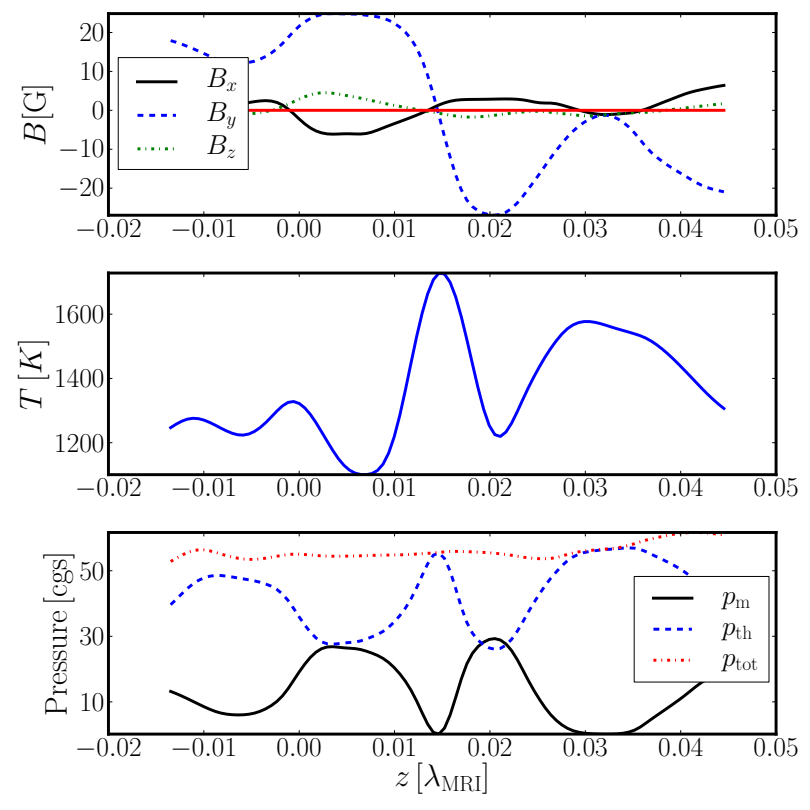

FIG. 8. - Data from a radial-vertical slice of the current sheet at (0.03 AU, $0.015 \mathrm{AU}$ ) in the highest resolution Pencil Code run at the same time as Figure 6. Vertical line cut through the current sheet. Top panel: magnetic field ( $\boldsymbol{x}$ in black, solid; $\boldsymbol{y}$ in blue, dashed; $\boldsymbol{z}$ in green, dash-dotted). Note the anti-correlation of $B_{x}$ and $B_{y}$ as the latter is the product of shearing the former. Middle panel: temperature. Bottom panel: magnetic pressure (black, solid), thermal pressure (blue, dashed), and total pressure(red, dash-dotted). This shows the degree of pressure balance across the current sheet.

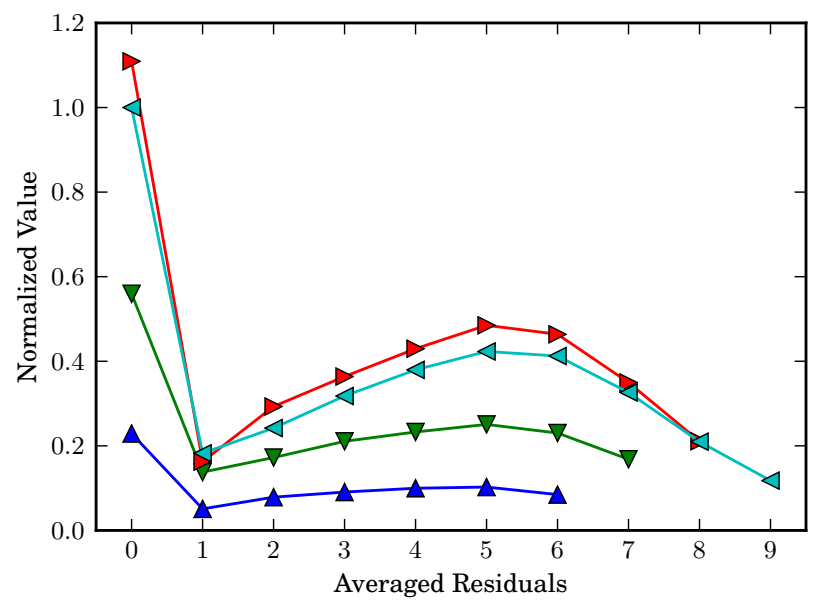

FIG. 9.- Time-averaged multiresolution decomposition of $|\boldsymbol{J}|^{2}$ in cubical Pencil Code runs. On the $x$-axis, 0 is the $v^{0}$ residual, and 1 through 9 are the $e^{1}$ to $e^{9}$ residuals corresponding to refined grid levels 1-9. All values are averages of absolute values over the mesh and over orbits $85-105$, and are normalized to the $v^{0}$ coefficient of the $512^{3}$ resolution run. The resolved peak in the residuals is at level 5. Base grid resolutions shown are $64^{3}$ (triangle up, blue), $128^{3}$ (triangle down, green), $256^{3}$ (triangle right, red), and $512^{3}$ (triangle left, cyan).

the Athena results are entirely consistent with the major result that current sheets can cause substantial local heating under the conditions we consider.

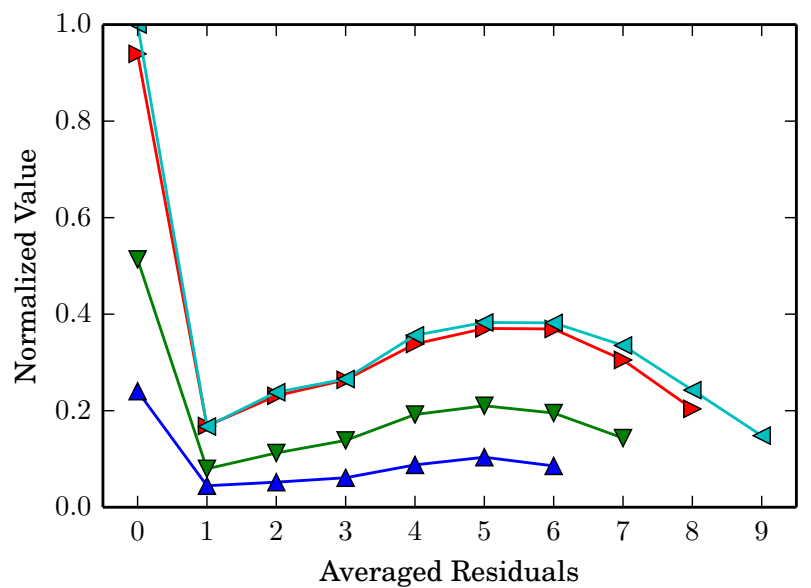

FIG. 10.- Time-averaged multiresolution decomposition of $|\boldsymbol{J}|^{2}$ in slab Pencil Code runs. The symbols and labels are the same as Figure 9
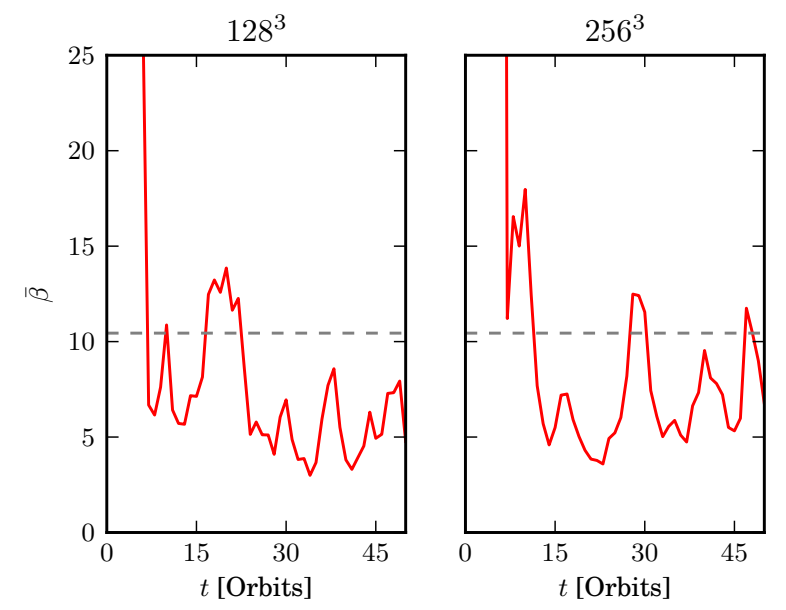

FIG. 11.- Time series of $\bar{\beta}$ for the Athena runs. Compare to Figure 2 top panels, the dashed gray reference line is the same as shown there.

\section{CURRENT SHEET HEATING}

Figures 4 and 6 clearly show that there are strong temperature variations in unstratified, net-vertical field MRI, not only in time but also in space. These large temperature fluctuations seem surprising given the modest overall $\bar{\beta}$ in the volume (Figure 2). The primary reason for this surprising behavior is that our magnetic energy is concentrated in large-scale azimuthal structures, which we call magnetic field bundles. These interact to generate thin current sheets with large horizontal extents that dissipate the magnetic energy. In this section we compute the temperature variations expected in this situation, both for analytical understanding of our results, and to extend the parameter space of their applicability.

\subsection{Adiabatic Compression}

The comparatively short perpendicular length scale of these current sheets implies that as long as $\beta \geq 1$, structures that are smaller than a scale height $H$ and survive 

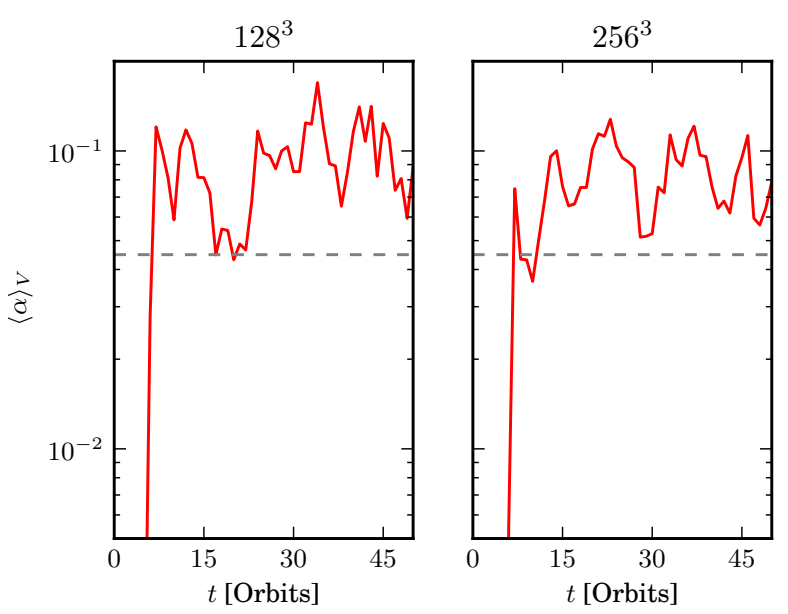

FIG. 12.- Time series of $\langle\alpha\rangle_{V}$ for the Athena runs. Compare to Figure 2, bottom panels, the dashed gray reference line is the same as shown there.
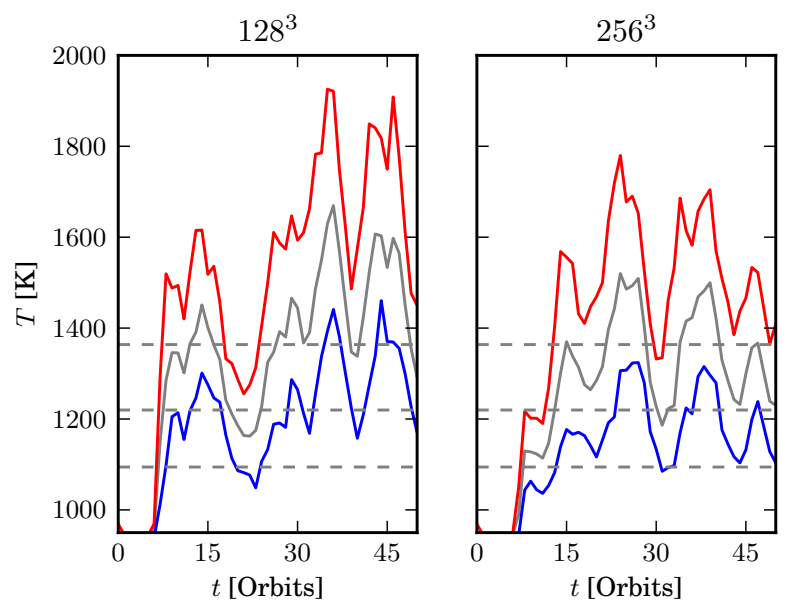

FIG. 13. - 90th percentile (red), 50th percentile (gray) and 10th percentile (blue) temperatures for the Athena runs. Compare to Figure 4 bottom panels, dashed gray lines are the same reference values shown there.

for at least an orbital timescale $\Omega^{-1}$ should be in near pressure equilibrium with the bounding magnetic field bundles. Accordingly, we can estimate the temperature variation by assuming that adiabatic compression maintains pressure equilibrium between the current sheet and the surrounding magnetic field bundles. Outside of the current sheet, the pressure is altered by both the growth of the magnetic field during the formation of the bundles and adiabatic expansion into the current sheet, while inside the current sheet the pressure is altered solely by adiabatic compression.

If we assume a sinusoidally varying magnetic field, and the maximum value of the magnetic pressure is small enough that we can expand linearly, then the adiabatic compression in the current sheets is equal to the adiabatic expansion outside the current sheets. Thus, if $\rho_{0}$, $T_{0}$ and $p_{0}$ are the density, temperature and pressure in the disk before the generation of the field bundles, the pressure $p_{1}$ of the system after magnetic field growth and pressure equilibration occurs will be

$$
p_{1}=p_{0}+\delta p_{\text {th }}=p_{0}-\delta p_{\text {th }}+\delta p_{m}
$$

where $\delta p_{m} \simeq p_{0} / \beta_{p}$ is the maximum magnetic pressure increase, $\beta_{p}$ is the minimum plasma $\beta$ associated with the peak magnetic field, and $\delta p_{\text {th }}$ is the amplitude of the thermal pressure variation. This implies that

$$
\delta p_{\mathrm{th}}=\frac{p_{0}}{2 \beta_{p}}
$$

The temperature variation between the magnetized regions and the current sheets is

$$
\frac{\delta T}{T_{0}}=\left(\frac{p_{0}+\delta p_{\mathrm{th}}}{p_{0}-\delta p_{\mathrm{th}}}\right)^{(\gamma-1) / \gamma}-1 \simeq \frac{\gamma-1}{\gamma} \frac{1}{\beta_{p}} .
$$

For our value of $\gamma=1.5$, this reduces to $\delta T / T_{0} \simeq$ $1 /\left(3 \beta_{p}\right)$.

Equation 19 implies that strong magnetic fields will always generate significant point-to-point temperature fluctuations through adiabatic compression alone, but this effect alone clearly is insufficient to explain the simulated behavior if $\beta_{p} \sim \bar{\beta} \sim 10$. However, there are two further effects we must consider. Thermal diffusion will reduce the temperature variation, but resistive heating in the current sheet will increase it.

\subsection{Resistive Heating and Thermal Diffusion}

In this section, we analyze the time-dependent, resistive heating of current sheets for magnetic fields whose peak magnetic field is weak, with a minimum value of the magnetic to thermal pressure ratio $\beta_{p}^{-1} \ll 1$. We expect that the azimuthal magnetic field bundles that bound the sheets form from the amplification of radial field variations by orbital shear. Such a mechanism should result in anticorrelation of radial and azimuthal magnetic fields. The top panel of Figure 8 demonstrates that this is indeed the case in our simulation.

We can therefore approximate the system as an initial radial field that varies only as a function of $z$ and decays resistively. We do not include any variation in the $x$-direction as the current sheets are thin, extended structures. As the azimuthal field bundles that appear in the simulations span the full box in azimuth, we also do not include any variation in the $y$-direction. However, such $y$ direction variations could be an interesting study, as they would sharpen with the action of the background shear. That radial field is sheared by a flow $u_{y}=S x$ where $S=-3 \Omega_{0} / 2$ is the Keplerian shear. The shear then generates an azimuthal field that also decays resistively. Under these conditions, the induction Equation (5) becomes (Brandenburg \& Subramanian 2005):

$$
\begin{aligned}
\frac{\partial B_{x}}{\partial t} & =\eta \frac{\partial^{2} B_{x}}{\partial z^{2}} \\
\frac{\partial B_{y}}{\partial t} & =-\frac{3 \Omega_{0}}{2} B_{x}+\eta \frac{\partial^{2} B_{y}}{\partial z^{2}}
\end{aligned}
$$

Assuming an initial radial field of $B_{x}=B_{0} \sin (k z)$, and no initial azimuthal field, the time evolution of the mag- 
netic field is

$$
\begin{aligned}
& B_{x}(t)=B_{0} \exp (-t / \tau) \sin (k z) \\
& B_{y}(t)=-\frac{3 \Omega_{0} t}{2} B_{x}=-B_{0}\left(\frac{3 \Omega_{0} t}{2}\right) \exp (-t / \tau) \sin (k z),
\end{aligned}
$$

where $\tau \equiv 1 /\left(\eta k^{2}\right)$ is the resistive decay time associated with the wavenumber $k$.

The peak azimuthal field occurs at time $t=\tau$. As long as the ratio of the resistive timescale $\tau$ to the shear timescale $S^{-1}$, namely $3 \Omega_{0} \tau / 2 \gg 1, B_{y}$ dominates the magnetic field, with a peak strength of

$$
B_{p}=\frac{3 \Omega_{0} \tau}{2 \exp (1)} B_{0} .
$$

In what follows, we consider only the heating due to this dominant azimuthal field, neglecting $B_{x}$ for all purposes other than feeding $B_{y}$.

We normalize the peak azimuthal field strength to the initial thermal pressure by defining the plasma- $\beta$ value for this peak azimuthal field with respect to the initial thermal pressure:

$$
\beta_{p} \equiv \frac{8 \pi p_{0}}{B_{p}^{2}} .
$$

We assume that $\beta_{p} \gg 1$, so that we can linearize in $\beta_{p}^{-1}$. In this limit, defining $\beta_{p}$ with respect to $p_{0}$ rather than the thermal pressure at the peaks of the perturbed magnetic field, where $\sin (k z)= \pm 1$, is equivalent to expanding first order in $\beta_{p}^{-1}$ because the thermal pressure perturbation does contribute at lowest order. We can use Equations (24) and (25) to rewrite the initial seed field strength in terms of $\beta_{p}$ :

$$
B_{0}^{2}=\frac{32 \pi \exp (2) p_{0}}{9 \Omega_{0}^{2} \tau^{2} \beta_{p}}
$$

In Gaussian cgs units, the Ohmic resistive energy dissipation is

$$
\frac{4 \pi \eta}{c^{2}} J^{2}=\frac{\eta}{4 \pi}(\nabla \times \boldsymbol{B})^{2} .
$$

Following the approximation that the magnetic field is dominated by the $B_{y}$ component yields

$$
\frac{4 \pi \eta}{c^{2}} J^{2}=\frac{2 \exp (2) p_{0}}{\beta_{p} \tau}\left(\frac{t}{\tau}\right)^{2} \exp (-2 t / \tau) \cos (k z)^{2} .
$$

Note that in the case of Ohmic resistivity, the magnetic energy dissipation is exactly $90^{\circ}$ out of phase spatially with the magnetic energy. This is neither the case with ambipolar diffusion (which scales with $\boldsymbol{J} \times \boldsymbol{B}$ ), nor with numerical dissipation.

Between time $t=0$ and $t$, Ohmic resistivity deposits an energy density

$$
E=\int_{0}^{t} d t^{\prime} \frac{\exp (2) p_{0}}{\beta_{p} \tau^{3}} t^{\prime 2} \exp \left(-2 t^{\prime} / \tau\right)(1+\cos (2 k z))
$$

into the gas. Using the relation for the thermal energy density $e=p /(\gamma-1)$, we can relate the total energy density $E$ deposited resistively to the resulting temperature fluctuation to first order in $\beta_{p}^{-1}: \delta T=\left[(\gamma-1) E / p_{0}\right] T_{0}$.

\subsubsection{Adiabatic Limit}

In the adiabatic limit, where the thermal diffusion coefficient $\mu \ll \eta$, and to first order in $\beta_{p}^{-1}$, there is neither advective nor diffusive energy transport. Therefore the highest point-to-point temperature variation occurs after all the magnetic energy has resistively dissipated. Then

$$
\begin{aligned}
\delta T & =2 \int_{0}^{\infty} d t^{\prime} \frac{(\gamma-1) T_{0} \exp (2)}{\beta_{p} \tau^{3}} t^{\prime 2} \exp \left(-2 t^{\prime} / \tau\right) \\
& =\frac{(\gamma-1) \exp (2)}{2 \beta_{p}} T_{0}, \quad \frac{\mu}{\eta} \ll 1
\end{aligned}
$$

where the factor of $\sin (\pi / 2)-\sin (3 \pi / 2)=2$ comes from taking the difference between the hottest and coldest points.

\subsubsection{Intermediate $\tau_{E}=\tau / 2$ Case}

An analytical solution is possible in the particular case that the thermal diffusion has a timescale $\tau_{E}=$ $1 /\left(4 \mu k^{2}\right)=\tau / 2$, then Equation 29) gives:

$$
\frac{\partial \delta T}{\partial t}=\frac{2(\gamma-1) T_{0} \exp (2)}{\beta_{p} \tau^{3}} t^{2} \exp (-2 t / \tau)-\frac{\delta T}{\tau_{E}} .
$$

In computing this solution note that the temperature fluctuation has wavenumber $2 k$ and Equation (32) can be solved analytically, becoming

$$
\delta T=\frac{(\gamma-1) \exp (2) t^{3} \exp \left(-t / \tau_{E}\right)}{12 \tau_{E}^{3} \beta_{p}} T_{0},
$$

which has a maximum for $t=3 \tau_{E}=1.5 \tau$ of

$$
\delta T=\frac{9(\gamma-1)}{4 \exp (1) \beta_{p}} T_{0} .
$$

This underestimates the final result because we have ignored the adiabatic expansion of the magnetic field bundle due to the magnetic pressure, which also generates temperature variations that are first order in $\beta_{p}^{-1}$ and are spatially in phase with the resistive heating. However, that signal would have modestly diffused away by $t=3 \tau_{E}$.

\subsubsection{Fast Cooling Limit}

Finally, if the temperature diffusion is very fast, the largest temperature variation will occur at the time of fastest heating, or $t=\tau$, when $B=B_{p}$, and the thermal energy perturbation will be just $\left(4 \pi / c^{2}\right) \eta J^{2} \tau_{E}$. This means

$$
\begin{aligned}
\delta T & =\frac{2(\gamma-1)}{\beta_{p}} \frac{\tau_{E}}{\tau} T_{0} \\
& =\frac{(\gamma-1)}{2 \beta_{p}} \frac{\eta}{\mu} T_{0}, \quad \frac{\mu}{\eta} \gg 1 .
\end{aligned}
$$

Here the heating simply scales by the ratio of magnetic diffusion to thermal diffusion $\eta / \mu$.

\subsection{Comparison to Simulations}

In our simulation, we use temperature independent values of the resistivity $\eta \sim 9 \times 10^{14} \mathrm{~cm}^{2} \mathrm{~s}^{-1}$ (Table 1), and 
the thermal diffusivity $\mu \sim 1.8 \times 10^{14} \mathrm{~cm}^{2} \mathrm{~s}^{-1}$ (Equation (8)), so

$$
\begin{aligned}
& \tau_{E} k^{2}=\frac{1}{4 \mu}=1.4 \times 10^{-15} \mathrm{~s} \mathrm{~cm}^{-2} \\
& \tau k^{2}=\frac{1}{\eta}=1.1 \times 10^{-15} \mathrm{~s} \mathrm{~cm}^{-2} .
\end{aligned}
$$

This means that, for any $k$, the timescale $\tau_{E} \sim \tau$, so we have slightly slower cooling than the regime of Equation (34), even though we are not in the $\beta_{p}^{-1} \ll 1$ regime. However, if we do apply Equation (34) to our simulations where $\beta_{p} \approx 1$ in both geometries, then the predicted $\Delta T / T_{0}=0.42$. This agrees reasonably well with the results obtained in Figures 4 and 5 , given the differing $\beta_{p}$ regimes, especially considering that, as discussed in Section 5.2.2. Equation (34) neglects the heating in the current sheet due to the adiabatic expansion of the magnetic field bundles. This latter will be particularly important when the system enters the $\beta_{p} \approx 1$ regime during particularly energetic phases. Due to this, one would expect that Equation (34) underestimates the heating which occurs in our simulations, as is the case.

\section{DISSIPATION COEFFICIENTS}

We have shown that the ratio of the resistivity to the thermal diffusion plays an important role in limiting the point-to-point temperature differences in protoplanetary disks. The MRI-criticality condition is $\Lambda_{0} \sim 0.1$, so in a disk with $c_{s} \propto R^{-1 / 4}$ (appropriate for the minimum mass solar nebula of Hayashi 1981, approximately the scaling of a passive irradiated disk, and reasonable for a constant- $\alpha$ disk), the critical resistivity scales with radius as

$$
\eta_{\text {crit }} \propto \beta_{\text {init }}^{-1} H^{2} \Omega \propto R^{1},
$$

where we have assumed a constant initial $\beta$ for the seed field. Radiative thermal diffusivity scales as

$$
\mu \propto \frac{T^{3}}{\rho^{2}} \propto R^{3}
$$

where we have assumed that the surface density scales with $\Sigma \propto R^{-1}$ of a constant- $\alpha$ disk with the given temperature scaling (Dullemond et al. 2007), and we have neglected the sublimation of dust grains. Note that letting the temperature vary with radius independently of $\eta$ implicitly assumes non-thermal ionization.

We chose our parameters to put the outer edge of the inner MRI active zone at our location of $1 \mathrm{AU}$. Given the radial scalings above, we can see that in the inner disk the resistivity at the outer edge of the thermally-ionized MRI active zone will usually dominate over thermal diffusion when the temperature is just adequate to ionize the gas sufficiently to the critical value for the MRI. Further, the difference between the power-laws in Equations 39 and (40) is large enough that this conclusion is robust against modest changes to the disk model. However, that analysis becomes unimportant once the temperature is high enough for the short-circuit instability to start, as it causes resistivity and opacity to depend strongly on temperature.

Beyond the dead zone, in the outer MRI-active regions of a protoplanetary disk, the non-thermal ionization relies on a low absorption column for cosmic rays. This implies a lower surface density, and hence a faster thermal diffusion than the inner MRI-active region. Therefore, when considering only the constant Ohmic resistivity considered in this paper, the temperature variations will be much smaller than those seen in the inner, thermally ionized MRI-active region of the disk, following Equation (36). If the ionization has a strong temperature dependence, as occurs at the metal lines suggested by Dzyurkevich et al. (2013), where ionization is dominantly controlled by the adsorption of ions onto grain surfaces, then the short-circuit instability of Hubbard et al. (2012) may modify the behavior of current sheets, leading to larger heating.

\section{DISCUSSION}

We have shown that, under reasonable parameters, the MRI can generate strong temperature variations across small regions. This has broad implications, from the dynamics of the MRI itself through changes in the gas density, through more indirect effects on the MRI such as the short-circuit instability, to effects that go beyond the MRI entirely, such as thermally processing solid material in protoplanetary disks. It is clear from our results that even minimal MRI activity can produce adequate temperature variations to trigger the short-circuit instability in regions with temperatures close to the thermal ionization regime.

\subsection{Caveats}

The generalizability of this result beyond the regime of our unstratified, net vertical field simulation does remain to be confirmed. In particular, including stratification could allow export of magnetic energy vertically through buoyancy; while net-vertical field MRI is relatively violent, so that relaxing these constraints might lead to weaker current sheets, providing less heating.

We have further demonstrated that resolving the current sheet structure of the MRI is a major undertaking. Even though we plausibly resolved the time-and-volume averaged magnetic field strength and stresses with only $128^{3}$ simulations (about 26 zones/scale height), the heating terms were only beginning to be resolved at $256^{3}$ (or about 50 zones/scale height). Further, this resolution requirement was for nearly the highest resistivity that would still allow the MRI with the fastest growing wavelength contained in the thin aspect ratio box height. Lower levels of resistivity will require even higher resolutions.

We employed a unstratified shearing box model, which limits the manner in which the large scale cooling of the disk can be taken into account. Within this framework, we chose the fastest consistent value for the cooling timescale. A vertically global model with full radiative transfer may have effectively slower thermal relaxation at the midplane, and therefore the temperature fluctuations produced in our model may be underestimates.

A significant difference between the case treated in our simulations and an actual protoplanetary disk is that we allow neither $\eta$ nor $\kappa$ to vary with temperature. Accordingly, because our simulations already show strong temperature fluctuations, we can state that any MRI simulations with temperature-independent resistivity and opacity in the temperature range we consider are not self-consistent. Our one-dimensional results on 
the short circuit instability (McNally et al. 2013) suggest that substantially stronger Huctuations would occur if a temperature-dependent resistivity and opacity are included.

For our simulations to progress, we must choose initial conditions that are MRI unstable. Awkwardly, as the MRI develops it heats the box. Were we to combine physically motivated, temperature-dependent resistivity with an initial condition that was marginally unstable to the MRI, the rising temperatures and falling resistivities would take the MRI away from marginal instability. Indeed, initially MRI-stable regions have been seen to become unstable as the turbulent disk heats (Latter \& Balbus 2012, Faure et al. 2014). Such radial variations cannot be captured by a local shearing box model, so the method used in this paper has a limited ability to treat the dynamically evolving region at the potentially migrating, certainly self-determining inner edge of the dead zone.

\subsection{Consequences}

Our result further supports the hypothesis that magnetic dissipation in general, and the short-circuit instability in particular may be responsible for the deduced thermal histories of calcium-aluminum rich inclusions (CAIs) and chondrules, as suggested by Hubbard et al. (2012) and McNally et al. (2013).

Several classes of igneous CAIs, particularly compact Type A, Type B, and Type C CAIs, appear to have been briefly heated back over their melting temperatures at some time after their initial condensation (Stolper \& Paque 1986, Scott \& Krot 2005). Being formed of the most refractory minerals, CAls may trace the inner, warm, and hence MRI-active, regions of the protoplanetary disk where temperature fluctuations generated by current sheets are unavoidable. Chondrules are made of less refractory material than CAIs, and so their thermal histories are often less extreme than those of remelted CAIs (e.g. Ebel 2006). However, they still require heating to temperatures where thermal ionization is easily capable of allowing the MRI to act.

Even in regions where the MRI is active but where the ionization does not sharply increase with temperature as required for the short-circuit instability, the temperature fluctuations we have found could provide a route to annealing silicates at more modest temperatures than are required to melt chondrules or CAIs (Sargent et al. 2009).

The scale of the temperature variations seen suggests that significant hysteresis may be possible in MRI activity. Once MRI begins, it may be able to provide the ionization to sustain itself even if the equilibrium disk becomes too neutral for it to continue (Latter \& Balbus 2012; Faure et al. 2014). The strongly time varying accretion rates of protoplanetary disks, for example as seen in FU Orionis type events (Hartmann \& Kenyon 1996), predict, and can be explained by, the inner active zone extending radially outwards (Armitage et al. 2001). If MRI activity is adequate to generate local hot regions with low enough resistivity to support the MRI, the MRI might be active even in regions where the median resistivity is too high to allow MRI.

This is a similar mechanism to that suggested by Inutsuka \& Sano (2005), although we favor current-driven heating resulting in thermal ionization rather than relying on direct non-thermal ionization by electrons accelerated in the current sheets. However, the end result, that current sheets may have a significantly lower resistivity, was explored by Muranushi et al. (2012) (see also erratum Muranushi et al. 2013) who found that some regions of what would otherwise be the dead zone can maintain an MRI active state by such a mechanism. Unlike the electron acceleration mechanism of Inutsuka \& Sano (2005), resistively heated current sheets can be expected to be most effective at maintaining MRI when the disk is massive, as this regime has the lowest thermal diffusion.

Another consequence of temperature variations is the broadening of ice lines. If the background temperature varies with radius as $R^{-1 / 2}$, then $20 \%$ temperature variations will broaden the ice-line to $\sim 40 \%$ of its "mean" position. A significant amount of material would then be repeatedly evaporated and recondensed (Ros \& Johansen 2013). This effect is significant even with quite small temperature variations: $4 \%$ temperature variations will still leave $8 \%$ broadening, producing an annulus with a width of a few local scale heights. The accretion crossing timescale for such an annulus exceeds $\alpha^{-1}$, long enough for material within it to be processed and reprocessed. Multiple rounds of evaporation and condensation are unlikely to result in the same grain porosity, and hence collision resilience, as direct collisional growth, so larger grain sizes could be reached than those expected from collisional growth, as computed, for example, by Windmark et al. (2012).

We thank D. Ebel for useful discussions, and G. Lesur for a constructive referee report that helped generalize our results. Supercomputing resources at Jülich Supercomputing Centre (PRACE project "Protostars: From Molecular Clouds to Disc Microphysics") and at $\mathrm{DeIC} / \mathrm{KU}$ in Copenhagen are gratefully acknowledged. The research leading to these results has received funding from the People Programme (Marie Curie Actions) of the European Union's Seventh Framework Programme (FP7/2007-2013) under REA grant agreement 327995 (C.P.M.), and the U.S. National Science Foundation under CDI grant AST08-35734 and AAG grant AST1009802 (A.H. and M.-M.M.L.).

\section{REFERENCES}

Armitage, P. J., Livio, M., \& Pringle, J. E. 2001, MNRAS, 324, 705

Balbus, S. A., \& Hawley, J. F. 1998, Rev. Mod. Phys., 70, 1

Blaes, O., Hirose, S., \& Krolik, J. H. 2007, ApJ, 664, 1057

Bodo, G., Mignone, A., Cattaneo, F., Rossi, P., \& Ferrari, A. 2008, A\&A, 487, 1
Brandenburg, A., \& Dobler, W. 2002, Computer Physics Communications, 147, 471

Brandenburg, A., Nordlund, A., Stein, R. F., \& Torkelsson, U. 1995, ApJ, 446, 741

Brandenburg, A., Nordlund, A., Stein, R. F., \& Torkelsson, U. 1996, ApJ, 458, L45

Brandenburg, A., \& Subramanian, K. 2005, Phys. Rep., 417, 1 
Cowley, S. C., Longcope, D. W., \& Sudan, R. N. 1997, Phys. Rep., 283, 227

Dullemond, C. P., Hollenbach, D., Kamp, I., \& D'Alessio, P. 2007, Protostars and Planets V, 555

Dzyurkevich, N., Turner, N. J., Henning, T., \& Kley, W. 2013, ApJ, 765, 114

Ebel, D. S. 2006, Condensation of Rocky Material in Astrophysical Environments, ed. D. S. Lauretta \& H. Y. McSween, 253-277

Faure, J., Fromang, S., \& Latter, H. 2014, A\&A, 564, A22

Flaig, M., Kissmann, R., \& Kley, W. 2009, MNRAS, 394, 1887

Fleming, T. P., Stone, J. M., \& Hawley, J. F. 2000, ApJ, 530, 464

Flock, M., Fromang, S., González, M., \& Commerçon, B. 2013, A\&A, 560, A43

Harten, A. 1994, Multiresolution Representation of Cell-Averaged Data, Tech. Rep. CAM Report 94-21, Department of Applied Mathematics, University of California Los Angles

Hartmann, L., \& Kenyon, S. J. 1996, ARA\&A, 34, 207

Hayashi, C. 1981, Progress of Theoretical Physics Supplement, 70,35

Hirose, S., Krolik, J. H., \& Stone, J. M. 2006, ApJ, 640, 901

Hirose, S., \& Turner, N. J. 2011, ApJ, 732, L30

Hubbard, A., McNally, C. P., \& Mac Low, M.-M. 2012, ApJ, 761, 58

Inutsuka, S.-i., \& Sano, T. 2005, ApJ, 628, L155

Jin, L. 1996, ApJ, 457, 798

King, A. R., \& Pringle, J. E. 2010, MNRAS, 404, 1903

Krolik, J. H., Hirose, S., \& Blaes, O. 2007, ApJ, 664, 1045

Latter, H. N., \& Balbus, S. 2012, MNRAS, 424, 1977

McNally, C. P., Hubbard, A., Mac Low, M.-M., Ebel, D. S., \& D'Alessio, P. 2013, ApJ, 767, L2
Muranushi, T., Okuzumi, S., \& Inutsuka, S.-i. 2012, ApJ, 760, 56 Muranushi, T., Okuzumi, S., \& Inutsuka, S.-i. 2013, ApJ, 771, 138 Parker, E. N. 1972, ApJ, 174, 499

Parker, E. N. 1994, Spontaneous current sheets in magnetic fields : with applications to stellar x-rays. International Series in Astronomy and Astrophysics, Vol. 1. New York : Oxford University Press, 1994., 1

Pessah, M. E., \& Chan, C.-K. 2008, ApJ, 684, 498

Rice, W. K. M., Paardekooper, S.-J., Forgan, D. H., \& Armitage, P. J. 2014, MNRAS, 438, 1593

Ros, K., \& Johansen, A. 2013, A\&A, 552, A137

Sano, T., \& Miyama, S. M. 1999, ApJ, 515, 776

Sargent, B. A., Forrest, W. J., Tayrien, C., McClure, M. K., Watson, D. M., Sloan, G. C., Li, A., Manoj, P., Bohac, C. J., Furlan, E., Kim, K. H., \& Green, J. D. 2009, ApJS, 182, 477

Scott, E. R. D., \& Krot, A. N. 2005, in Astronomical Society of the Pacific Conference Series, Vol. 341, Chondrites and the Protoplanetary Disk, ed. A. N. Krot, E. R. D. Scott, \& B. Reipurth, 15

Shakura, N. I., \& Sunyaev, R. A. 1973, A\&A, 24, 337

Stolper, E., \& Paque, J. M. 1986, Geochim. Cosmochim. Acta, 50,1785

Stone, J. M., \& Gardiner, T. A. 2010, ApJS, 189, 142

Stone, J. M., Gardiner, T. A., Teuben, P., Hawley, J. F., \& Simon, J. B. 2008, ApJS, 178, 137

Turner, N. J. 2004, ApJ, 605, L45

Turner, N. J., Stone, J. M., Krolik, J. H., \& Sano, T. 2003, ApJ, 593,992

Windmark, F., Birnstiel, T., Ormel, C. W., \& Dullemond, C. P. 2012, A\&A, 544, L16 